\title{
Current Fluctuations for Independent Random Walks in Multiple Dimensions
}

\author{
Rohini Kumar
}

Received: 25 October 2009 / Revised: 7 July 2010 / Published online: 6 October 2010

(C) The Author(s) 2010. This article is published with open access at Springerlink.com

\begin{abstract}
Consider a system of particles evolving as independent and identically distributed (i.i.d.) random walks. Initial fluctuations in the particle density get translated over time with velocity $\vec{v}$, the common mean velocity of the random walks. Consider a box centered around an observer who starts at the origin and moves with constant velocity $\vec{v}$. To observe interesting fluctuations beyond the translation of initial density fluctuations, we measure the net flux of particles over time into this moving box. We call this the "box-current" process.

We generalize this current process to a distribution-valued process. Scaling time by $n$ and space by $\sqrt{n}$ gives current fluctuations of order $n^{d / 4}$ where $d$ is the space dimension. The scaling limit of the normalized current process is a distribution-valued Gaussian process with given covariance. The limiting current process is equal in distribution to the solution of a given stochastic partial differential equation which is related to the generalized Ornstein-Uhlenbeck process.
\end{abstract}

Keywords Independent random walks · Hydrodynamic limit - Current fluctuations · Distribution-valued process · Generalized Ornstein-Uhlenbeck process

Mathematics Subject Classification Primary 60K35 - 60F10 - Secondary 60F17 . 60G15

\section{Introduction}

In a system of independent random walks in dimension $d \geq 1$, the hydrodynamic limit of particle density, under Euler scaling, satisfies the scalar conservation law

$$
\partial_{t} u(x, t)+\nabla_{x} \cdot f(u(x, t))=0,
$$

R. Kumar $(\bowtie)$

Statistics and Applied Probability, UCSB, Santa Barbara, CA 93106, USA

e-mail: kumar@pstat.ucsb.edu 
with $u(x, 0)=u_{0}(x)$ as the initial particle density and where $t \geq 0, x \in \mathbb{R}^{d}$ and $f(u)=u \vec{v}, \vec{v}$ being the mean drift of the random walks. What we mean by this hydrodynamic limit is the following. If we start with a sequence of initial particle configurations $\left\{\eta_{0}^{n}(m): m \in \mathbb{Z}^{d}\right\}$ satisfying

$$
\frac{1}{n^{d}} \sum_{x \in n A \cap \mathbb{Z}^{d}} \eta_{0}^{n}(x) \longrightarrow \int_{A} u_{0}(x) d x \quad \text { in probability }
$$

where $A \subset \mathbb{R}^{d}$ is a bounded box, then the particle configurations at subsequent times, $\eta_{t}^{n}(\cdot)$, satisfy

$$
\frac{1}{n^{d}} \sum_{x \in n A \cap \mathbb{Z}^{d}} \eta_{n t}^{n}(x) \longrightarrow \int_{A} u(x, t) d x \quad \text { in probability }
$$

where $u(x, t)$ satisfies (1.1) with initial condition $u(x, 0)=u_{0}(x)$. This hydrodynamic limit picture indicates that on time and space scales of $O(n)$ we see shifts in the particle profile along characteristic lines of (1.1) given by $n y+n \vec{v} t, y \in \mathbb{R}^{d}$. To capture diffusive fluctuations about these characteristic lines, we consider a box of radius $O(\sqrt{n})$ moving with the characteristic velocity $\vec{v}$, and we look at the net inward flux of particles across the boundary of this moving box. The measurement of this net flux of particles gives us what we call the 'box-current' process. We generalize this box-current process to a distribution-valued process. In Sect. 2.3, we give a precise definition of these current processes and explain the connection between the current process and fluctuations about the hydrodynamic limit.

The question we resolve in this paper is the size and the distribution of the scaling limit of current fluctuations. It turns out that the current process scaled by $n^{-d / 4}$, where $n$ is the scaling parameter, gives us Gaussian scaling limits. The limiting distribution-valued current process can also be attained as the solution of a stochastic partial differential equation. An alternate representation of the limiting current process as the solution of a stochastic differential equation is given in Sect. 2.5. The limiting current process is expressed as the sum of two independent stochastic integrals, where one of the integrals is a generalized Ornstein-Uhlenbeck process.

In 1985, current fluctuations in a system of independently moving particles was studied in [2] in connection with the asymptotic behavior of trajectories in an infinite particle system with collisions. Seppäläinen, in [10], found the scaling limit of current across characteristics in the one-dimensional system of asymmetric independent random walks. His work was extended in [7] where shifts in characteristics at the diffusive scale were allowed. In both cases current fluctuations were of order $n^{1 / 4}$, where $n$ was the scaling factor, and Gaussian scaling limits were obtained. In [7], a representation of the limiting current process as the sum of two stochastic integrals was given for the one-dimensional case which agrees with the results in Sect. 2.5 of this paper. While in the one-dimensional system of independent random walks there is a connection between the position of a tagged particle under elastic collisions and a current process (see [2]), there is no such obvious connection in higher dimensions.

Equilibrium fluctuations in particle density, in a system of independent Markovian particles in multiple dimensions, was studied by Anders Martin-Löf in 1976 [8]. Our 
results about current fluctuations hold in the equilibrium as well as non-equilibrium case, under the assumption that the initial distribution of number of particles at each site on the lattice $\mathbb{Z}^{d}$ is i.i.d. It is interesting to note that in the absence of fluctuations in the initial particle configuration (i.e. if we start with a deterministic initial configuration), our limiting current process is a generalized Ornstein-Uhlenbeck process. Generalized Ornstein-Uhlenbeck processes appear as the fluctuation limits of several infinite particle systems in the literature, for example see Chap. 11 of Kipnis and Landim [1, 4, 6, 8].

Fluctuation results for other asymmetric models in multiple dimensions can be found in the literature. We mention some of these. In multiple dimensions, equilibrium fluctuations for a tagged particle in asymmetric exclusion processes and in asymmetric zero range processes have been studied in [11, 14] and [13] respectively. The diffusion coefficient for the two-dimensional asymmetric simple exclusion process was studied in [15]. In [12], Sethuraman proves superdiffusivity of occupation-time variance in the two-dimensional asymmetric exclusion process.

In this paper we extend the results of [10] and [7] to multiple dimensions. We also generalize their results from a simple real-valued current process to a distributionvalued current process. Our proof parallels the proof in [10] and [7] in several places. Some significant differences arise in the treatment of a distribution-valued process. As is typical for process-level distributional limits, our proof is in two stages: first, we prove convergence of finite-dimensional distributions and second, we prove tightness of the current process. We omit parts of the proof that are similar to parts in [7] and refer the reader to [7] for details.

This paper is structured as follows. Section 2 contains a description of the independent random walks model, the current process and a statement of our results. The stochastic partial differential equation satisfied by the limiting current process and its solution are also mentioned here. In Sect. 3 we find the finite-dimensional distribution limits of the process. We prove tightness of the process and complete the proof in Sect. 4.

\section{Model and Results}

\subsection{Independent Random Walks Model}

Let $d \geq 1$ denote the space dimension. We start with an initial configuration of particles on $\mathbb{Z}^{d}$ denoted by $\left\{\eta_{0}(x): x \in \mathbb{Z}^{d}\right\}$, i.e. $\eta_{0}(x)$ is the number of particles at site $x$ at time 0 . These particles evolve like independent, identically distributed (i.i.d.) continuous time random walks. Let $X_{m, j}(t), m \in \mathbb{Z}^{d}, j=1, \ldots, \eta_{0}(m)$ denote the position at time $t$ of the $j$-th random walk starting at site $m$. Let $\left\{\eta_{t}(x): x \in \mathbb{Z}^{d}\right\}$ denote the occupation variables at time $t$ i.e. $\eta_{t}(x)$ is the number of random walks (particles) at site $x$, at time $t$. Clearly,

$$
\eta_{t}(x):=\sum_{m \in \mathbb{Z}^{d}} \sum_{j=1}^{\eta_{0}(m)} \mathbf{1}\left\{X_{m, j}(t)=x\right\},
$$

$x \in \mathbb{Z}^{d}$. 
The common jump rates of the random walks are given by the probability kernel $\left\{p(x): x \in \mathbb{Z}^{d}\right\}$ where we assume

$$
\sum_{x \in \mathbb{Z}^{d}} p(x)=1
$$

The common transition probabilities of the random walks is given by

$$
P\left(X_{m, j}(s+t)=y \mid X_{m, j}(s)=x\right)=\sum_{k=0}^{\infty} \frac{e^{-t} t^{k}}{k !} p^{(k)}(y-x)
$$

where

$$
p^{(k)}(z)=\sum_{x_{1}+x_{2}+\cdots+x_{k}=z} p\left(x_{1}\right) \cdot\left(x_{2}\right) \cdots p\left(x_{k}\right)
$$

is the $k$-fold convolution of the kernel $p(x)$.

We make the following assumptions.

Assumption 2.1 The initial occupation variables $\left\{\eta_{0}(m): m \in \mathbb{Z}^{d}\right\}$ are i.i.d. random variables with exponential moments i.e.

$$
E e^{\theta \eta_{0}(m)}<\infty
$$

for some $\theta>0$. Let $\rho_{0}:=E \eta_{0}(m)$ and $v_{0}:=\operatorname{Var} \eta_{0}(m)$.

Assumption 2.2 Given $\left\{\eta_{0}(m): m \in \mathbb{Z}^{d}\right\}$, the evolution of the random walks $\left\{X_{m, j}(t)-X_{m, j}(0): m \in \mathbb{Z}^{d}, j=1, \ldots, \eta_{0}(m)\right\}$ are i.i.d. continuous time random walks on $\mathbb{Z}^{d}$ starting at the origin, independent of $\eta_{0}(\cdot)$. We can assume without loss of generality that the common jump probability kernel $\left\{p(x): x \in \mathbb{Z}^{d}\right\}$ is not supported on a hyperplane in $\mathbb{R}^{d}$. Let

$$
\vec{v}:=\sum_{x} x p(x)
$$

denote average velocity of the random walks. Define a $d \times d$ matrix a, related to the second moments of the random walks, as

$$
\mathbf{a}_{i, j}:=\sum_{x=\left(x_{1}, \ldots, x_{d}\right) \in \mathbb{Z}^{d}} x_{i} x_{j} p(x),
$$

and choose matrix $\kappa$ such that

$$
\kappa \kappa^{T}=\mathbf{a} .
$$

\section{Assumption 2.3}

$$
\sum_{x \in \mathbb{Z}^{d}} e^{\delta|x|} p(x)<\infty
$$

for some $\delta>0$. 


\subsection{Some Standard Notation}

We define the norm of $x=\left(x_{1}, \ldots, x_{d}\right) \in \mathbb{R}^{d}$ as

$$
|x|:=\max _{1 \leq i \leq d}\left|x_{i}\right|
$$

For $x \in \mathbb{R}$, define

$$
[x]:= \begin{cases}\text { largest integer } \leq x, & \text { if } x \geq 0 \\ \text { smallest integer } \geq x, & \text { if } x \leq 0 .\end{cases}
$$

Define

$$
[x]:=\left(\left[x_{1}\right], \ldots,\left[x_{d}\right]\right)
$$

for $x=\left(x_{1}, x_{2}, \ldots, x_{d}\right) \in \mathbb{R}^{d}$.

Let

$$
\partial^{\alpha}:=\left(\frac{\partial}{\partial x_{1}}\right)^{\alpha_{1}} \cdots\left(\frac{\partial}{\partial x_{d}}\right)^{\alpha_{d}}
$$

for a multi-index $\alpha=\left(\alpha_{1}, \ldots, \alpha_{d}\right)$ with $\alpha_{i} \geq 0$. For any nonnegative integer $N$ and multi-index $\alpha=\left(\alpha_{1}, \ldots, \alpha_{d}\right), \alpha_{i} \geq 0$, define

$$
\|f\|_{(N, \alpha)}:=\sup _{x \in \mathbb{R}^{d}}\left\{(1+|x|)^{N}\left|\partial^{\alpha} f(x)\right|\right\} .
$$

Let $\mathcal{S}\left(\mathbb{R}^{d}\right)$ denote the space of Schwartz functions. Recall that this space of functions is defined as

$$
\mathcal{S}\left(\mathbb{R}^{d}\right):=\left\{f \in C^{\infty}\left(\mathbb{R}^{d}\right):\|f\|_{(N, \alpha)}<\infty \forall N, \alpha\right\}
$$

We denote the dual of the Schwartz space as $\mathcal{S}^{\prime}\left(\mathbb{R}^{d}\right)$. For any bounded subset $B$ of $\mathcal{S}\left(\mathbb{R}^{d}\right)$, let

$$
q_{B}(f):=\sup _{\phi \in B}|f(\phi)|, \quad f \in \mathcal{S}^{\prime}\left(\mathbb{R}^{d}\right) .
$$

Then $\left\{q_{B}\right\}$ is a family of semi-norms on $\mathcal{S}^{\prime}\left(\mathbb{R}^{d}\right)$ which defines the strong topology on $\mathcal{S}^{\prime}\left(\mathbb{R}^{d}\right)$. Fix $T>0$, let $D\left([0, T], \mathcal{S}^{\prime}\left(\mathbb{R}^{d}\right)\right)$ be the space of mappings from $[0, T]$ to $\mathcal{S}^{\prime}\left(\mathbb{R}^{d}\right)$ that are right continuous and have left-hand limits in the strong topology of $\mathcal{S}^{\prime}\left(\mathbb{R}^{d}\right)$.

2.3 The Current Process and Fluctuations About the Hydrodynamic Limit

Let

$$
B_{M}:=\left\{x \in \mathbb{R}^{d}:|x| \leq M\right\}
$$

be a box centered at the origin with radius $M$. Suppose this box is centered about an observer who starts at the origin and moves with constant velocity $\vec{v}$. We define 
the 'box-current process' as the net inward flux of particles across the boundary of this moving box, over time. Thus the box-current at time $t$ is simply the difference between the number of particles inside the box at time $t$ and the number of particles initially inside the box. Scaling space by $\sqrt{n}$ and time by $n$, we construct a sequence of scaled box-current processes given by

$$
\begin{aligned}
\xi_{n}\left(t, \mathbf{1}_{B_{M}}\right) & :=n^{-d / 4} \sum_{i \in B_{M \sqrt{n}}}\left\{\eta_{n t}(i+[n \vec{v} t])-\eta_{0}(i)\right\} \\
& =n^{-d / 4} \sum_{m \in \mathbb{Z}^{d}} \sum_{j=1}^{\eta_{0}(m)}\left[\mathbf{1}\left\{X_{m, j}(n t) \in B_{M \sqrt{n}}+[n \vec{v} t]\right\}-\mathbf{1}\left\{m \in B_{M \sqrt{n}}\right\}\right] .
\end{aligned}
$$

In words, $\xi_{n}\left(t, \mathbf{1}_{B_{M}}\right)$ measures the cumulative net current of particles into the box $B_{M \sqrt{n}}$ up to time $n t$ as the box moves with fixed velocity $\vec{v}$.

We generalize the above box-current process to a distribution-valued process by taking the weighted average of the differences $\left\{\eta_{n t}(i+[n \vec{v} t])-\eta_{0}(i)\right\}$, where the weights are given by values of a Schwartz function. For $\phi \in \mathcal{S}\left(\mathbb{R}^{d}\right)$ and $t \in[0, T]$, define

$$
\xi_{n}(t, \phi):=n^{-d / 4} \sum_{i \in \mathbb{Z}^{d}}\left\{\phi\left(\frac{i}{\sqrt{n}}\right)\left(\eta_{n t}(i+[n \vec{v} t])-\eta_{0}(i)\right)\right\} .
$$

This is our current process. Observe that $\xi_{n}(\cdot, \cdot) \in D\left([0, T], \mathcal{S}^{\prime}\left(\mathbb{R}^{d}\right)\right)$. We can rewrite $\xi_{n}(t, \phi)$ as

$$
\begin{aligned}
\xi_{n}(t, \phi) & n^{-d / 4} \sum_{i \in \mathbb{Z}^{d}} \phi\left(\frac{i}{\sqrt{n}}\right)\left\{\sum_{m \in \mathbb{Z}^{d}} \sum_{j=1}^{\eta_{0}(m)}\left[\mathbf{1}\left\{X_{m, j}(n t)=i+[n \vec{v} t]\right\}-\mathbf{1}\{m=i\}\right]\right\} \\
= & n^{-d / 4} \sum_{m \in \mathbb{Z}^{d}} \sum_{j=1}^{\eta_{0}(m)}\left[\phi\left(\frac{X_{m, j}(n t)-[n \vec{v} t]}{\sqrt{n}}\right)\left(\sum_{i \in \mathbb{Z}^{d}} \mathbf{1}\left\{X_{m, j}(n t)=i+[n \vec{v} t]\right\}\right)\right. \\
& \left.-\phi\left(\frac{m}{\sqrt{n}}\right)\right] \\
= & n^{-d / 4} \sum_{m \in \mathbb{Z}^{d}} \sum_{j=1}^{\eta_{0}(m)}\left[\phi\left(\frac{X_{m, j}(n t)-[n \vec{v} t]}{\sqrt{n}}\right)-\phi\left(\frac{m}{\sqrt{n}}\right)\right] .
\end{aligned}
$$

In the last line of (2.3) we are summing over all random walks and adding their contributions to the current. This is a more tractable form of the current process compared to its initial definition in (2.2) and we will use this definition henceforth for the current process.

As in [10], here too there is a connection between the current process and what the author in [10] termed the "second-order fluctuations" from the hydrodynamic limit. We explore this connection below. Let $(\Omega, \mathcal{F}, \mathcal{P})$ be the underlying probability 
measure space for our independent random walk process. By our assumptions, under $\mathcal{P}$, the initial occupation variables $\left\{\eta_{0}(m), m \in \mathbb{Z}^{d}\right\}$ are i.i.d. random variables and are independent of the evolution of the random walks $\left\{X_{m, j}(\cdot)-X_{m, j}(0): m \in \mathbb{Z}^{d}, j=\right.$ $\left.1, \ldots, \eta_{0}(m)\right\}$. Under these assumptions we get

$$
n^{-d / 2} \sum_{i \in \mathbb{Z}^{d}} \phi\left(\frac{i}{\sqrt{n}}\right) \eta_{n t}(i+[n \vec{v} t]) \stackrel{n \rightarrow \infty}{\longrightarrow} \rho_{0} \int_{x \in \mathbb{R}^{d}} \phi(x) d x \quad \text { in } L^{2}(\mathcal{P}) \text { for all } t \geq 0,
$$

where $E\left[\eta_{0}(m)\right]=\rho_{0}$. (For general initial conditions with $E\left[\eta_{0}^{n}(x)\right]=\rho_{0}\left(\frac{x}{\sqrt{n}}\right)$, the right hand side would be $\int \rho(x, t) \phi(x) d x$ where $\rho(x, t)$ is the solution of a diffusion equation with initial condition $\rho_{0}(\cdot)$. Since $\rho_{0}(x) \equiv \rho_{0}$ is a constant in our problem, we get $\rho(x, t) \equiv \rho_{0}$.)

We can express $\xi_{n}(t, \phi)$ as the difference of two terms:

$$
\begin{aligned}
\xi_{n}(t, \phi)= & n^{d / 4}\left[\left(n^{-d / 2} \sum_{i \in \mathbb{Z}^{d}} \phi\left(\frac{i}{\sqrt{n}}\right) \eta_{n t}(i+[n \vec{v} t])-\rho_{0} \int_{x \in \mathbb{R}^{d}} \phi(x) d x\right)\right. \\
& \left.-\left(n^{-d / 2} \sum_{i \in \mathbb{Z}^{d}} \phi\left(\frac{i}{\sqrt{n}}\right) \eta_{0}(i)-\rho_{0} \int_{x \in \mathbb{R}^{d}} \phi(x) d x\right)\right] .
\end{aligned}
$$

Using the observer as a frame of reference, the first term is the fluctuation from the hydrodynamic limit at time $t$ and the second term is the fluctuation from the hydrodynamic limit at time 0 . Thus, $\xi_{n}(t, \phi)$ is obtained by subtracting the initial fluctuations from the hydrodynamic limit from the fluctuations at time $t$ from the hydrodynamic limit, as seen by the observer, and scaling this difference by $n^{d / 4}$. The current process therefore looks at fluctuations beyond the rigid translation of fluctuations in the initial configuration along the characteristic line $y=x+\vec{v} t$.

\subsection{Results}

Recall that $\left\{p(x), x \in \mathbb{Z}^{d}\right\}$ denote the common jump probabilities of the random walks. Let $X(t)$ be a continuous time random walk starting at the origin, with jump probabilities $p(x)$. Henceforth, in this paper, $X(t)$ will always denote such a random walk. By the martingale central limit theorem (refer pp. 339-340 in [3]), we have

$$
\frac{X(n t)-[n \vec{v} t]}{\sqrt{n}} \Longrightarrow \kappa B(t)
$$

where $B(t)$ is standard d-dimensional Brownian motion. The probability density function of $\kappa B(t)$ is given by

$$
p_{t}(x) d x:=P(\kappa B(t) \in d x)=\frac{e^{-\frac{1}{2 t} \sum_{i, j} \mathbf{a}_{i, j}^{-1} x_{i} x_{j}}}{(2 \pi t)^{d / 2} \sqrt{\operatorname{det}(\mathbf{a})}} d x .
$$

(Note: Since $p(x)$ is not supported on a hyperplane in $\mathbb{R}^{d}$, a is positive definite.) 
Define

$$
\begin{aligned}
\sigma_{1}((s, \phi),(t, \psi)):= & \int_{\mathbb{R}^{d}} \int_{\mathbb{R}^{d}} \phi(y) \psi(z) p_{|t-s|}(z-y) d z d y \\
& -\int_{\mathbb{R}^{d}} \int_{\mathbb{R}^{d}} \phi(y) \psi(z) p_{t+s}(z-y) d z d y
\end{aligned}
$$

and

$$
\begin{aligned}
\sigma_{2}((s, \phi),(t, \psi)):= & \int_{\mathbb{R}^{d}} \int_{\mathbb{R}^{d}} \phi(y) \psi(z) p_{t+s}(z-y) d z d y \\
& -\int_{\mathbb{R}^{d}} \int_{\mathbb{R}^{d}} \phi(y) \psi(z) p_{t}(z-y) d z d y \\
& -\int_{\mathbb{R}^{d}} \int_{\mathbb{R}^{d}} \phi(y) \psi(z) p_{s}(z-y) d z d y \\
& +\int_{\mathbb{R}^{d}} \phi(x) \psi(x) d x
\end{aligned}
$$

where $p_{t}(x)$ is the probability density function of $\kappa B(t)$ given in (2.5).

Theorem 2.1 Under Assumptions 2.1-2.3, as $n \rightarrow \infty, \xi_{n}(\cdot, \cdot) \rightarrow \xi(\cdot, \cdot)$ in distribution in $D\left([0, T], \mathcal{S}^{\prime}\left(\mathbb{R}^{d}\right)\right)$ where $\xi(\cdot, \cdot)$ is a mean zero, distribution-valued Gaussian process with covariance function

$$
E \xi(s, \phi) \xi(t, \psi)=\rho_{0} \sigma_{1}((s, \phi),(t, \psi))+v_{0} \sigma_{2}((s, \phi),(t, \psi))
$$

Theorem 2.2 Under Assumptions 2.1-2.3, as $n \rightarrow \infty, \xi_{n}\left(\cdot, \mathbf{1}_{B_{M}}\right)$ converges in distribution in $D([0, T], \mathbb{R})$ to a mean zero Gaussian process with covariance as in $(2.8)$ where $\phi=\psi=\mathbf{1}_{B_{M}}$.

Example We calculate the covariance terms (2.6) and (2.7) for a simple example. Take $\phi \equiv \psi:=\mathbf{1}_{B_{M}}=\prod_{i=1}^{d} \mathbf{1}_{[-M, M]}$ and $\mathbf{a}=I_{d \times d}$. Let $\Phi_{u}(\cdot)$ denote the cumulative distribution function of a mean zero real-valued Gaussian random variable (i.e. $\left.\Phi_{u}(x):=\frac{1}{\sqrt{2 \pi u}} \int_{-\infty}^{x} e^{-\frac{x^{2}}{2 u}}\right)$ and define

$$
I_{u}=\left[2 M\left\{\Phi_{u}(2 M)-\Phi_{u}(-2 M)\right\}+2 \sqrt{\frac{u}{2 \pi}}\left(e^{\frac{-2 M^{2}}{u}}-1\right)\right]^{d} .
$$

Then

$$
\sigma_{1}((s, \phi),(t, \psi))=I_{|t-s|}-I_{t+s},
$$

and

$$
\sigma_{2}((s, \phi),(t, \psi))=I_{t+s}-I_{t}-I_{s}+(2 M)^{d}
$$


Remark 1 In the long-run, the current process is asymptotically a stationary Gaussian process. This can be observed by taking the long time asymptotic limit of the covariance terms (2.6) and (2.7).

Remark 2 If we start with an initial configuration where $\eta_{0}(\cdot)$ are i.i.d. Poisson $(\lambda)$ random variables, the system of independent random walks is in equilibrium and we get $\eta_{t}(\cdot)$ are i.i.d. Poisson $(\lambda)$ for all $t \geq 0$. Under this invariant distribution of particles, the limiting current process in [7] and [10] are fractional Brownian motion with Hurst parameter $1 / 4$. In the present paper, under this invariant distribution of particles, $\rho_{0}=v_{0}=\lambda$ and

$$
\begin{aligned}
E & {[\xi(t, \phi)-\xi(s, \psi)]^{2} } \\
& =E\left[\xi(t, \phi)^{2}+\xi(s, \phi)^{2}-2 \xi(t, \phi) \xi(s, \phi)\right] \\
& =\lambda\left[\int_{\mathbb{R}^{d}} \phi(x) \psi(x) d x-\int_{\mathbb{R}^{d}} \int_{\mathbb{R}^{d}} \phi(y) \psi(z) p_{t-s}(z-y) d z d y\right] .
\end{aligned}
$$

Thus, under the invariant distribution, $\xi(\cdot, \phi)$ has stationary increments, as $E(\xi(t, \phi)-\xi(s, \phi))^{2}$ is a function of $t-s$. The time-indexed process $\xi(\cdot, \phi)$ lacks the self-similarity property of one-parameter fBM. However, the process $\xi(t, \phi)$ satisfies the following self-similarity property:

$$
\xi\left(a t, \phi \circ \eta_{a}\right) \stackrel{d}{=} a^{d / 2} \cdot \xi(t, \phi), \quad \text { for } a>0,
$$

where $\eta_{a}(x)=\frac{x}{\sqrt{a}}, x \in \mathbb{R}^{d}$; this is evident from the form of the covariance terms (2.6) and (2.7).

The proof of both theorems involves two stages: first, showing convergence of finite-dimensional distributions and second, proving tightness of the sequence of processes. We use Mitoma's theorem [9] to prove tightness of $\left\{\xi_{n}(\cdot, \cdot)\right\} \subset$ $D\left([0, T], \mathcal{S}^{\prime}\left(\mathbb{R}^{d}\right)\right)$. According to Mitoma's theorem, it is sufficient to prove tightness for the sequence $\left\{\xi_{n}(\cdot, \phi)\right\} \subset D([0, T], \mathbb{R})$ for each $\phi \in \mathcal{S}\left(\mathbb{R}^{d}\right)$. We use the tightness criteria in [2] to prove tightness of the sequence $\left\{\xi_{n}(\cdot, \phi)\right\} \subset D([0, T], \mathbb{R})$. A description of Schwartz space $\mathcal{S}\left(\mathbb{R}^{d}\right)$, it's dual space $\mathcal{S}^{\prime}\left(\mathbb{R}^{d}\right)$ and Mitoma's theorem can be found in [5].

\subsection{Stochastic Integral Representation}

The limiting current process in Theorem 2.1 has the same distribution as the solution of the stochastic partial differential equation given below in (2.13).

Define operator $\mathcal{A}$ on $\mathcal{S}\left(\mathbb{R}^{d}\right.$ ) (the infinitesimal generator of $\kappa B(t)$ ) as

$$
\mathcal{A} \phi(x):=\frac{1}{2} \sum_{i, j=1}^{d} \mathbf{a}_{i, j} \partial_{i j} \phi(x) .
$$


Let $\left\{T_{t}, t \geq 0\right\}$ denote the semigroup associated to $\mathcal{A}$. Then

$$
T_{t}(\phi)(x)=\int_{\mathbb{R}^{d}} \phi(y) p_{t}(y-x) d y .
$$

Define covariance functions

$$
Q_{1}(\phi, \psi):=\int_{\mathbb{R}^{d}} \sum_{i, j=1}^{d} \mathbf{a}_{i, j} \partial_{i} \phi(x) \partial_{j} \psi(x) d x,
$$

and

$$
Q_{2}(\phi, \psi):=\int_{\mathbb{R}^{d}} \mathcal{A} \phi(x) \mathcal{A} \psi(x) d x .
$$

Consider the following spde,

$$
d Z(t, \cdot)=\sqrt{\rho_{0}} d W_{t}+\mathcal{A} Z(t, \cdot) d t+\sqrt{v_{0}} \mathcal{F}(\cdot) d t ; \quad Z(0, \cdot)=0,
$$

where $W_{t}$ is a centered $\mathcal{S}^{\prime}\left(\mathbb{R}^{d}\right)$-Wiener process with covariance $Q_{1}(\cdot \cdot),\{\mathcal{F}(\phi), \phi \in$ $\left.\mathcal{S}\left(\mathbb{R}^{d}\right)\right\}$ is a Gaussian random field on $\mathcal{S}\left(\mathbb{R}^{d}\right)$ with covariance $Q_{2}(\cdot, \cdot)$ and $\mathcal{F}$ and $W_{t}$ are independent of each other. We can write

$$
W_{t}^{\phi}=\int_{[0, t] \times \mathbb{R}^{d}} \nabla \phi(x) \cdot \kappa W_{1}(d t d x)
$$

where $W_{1}(t, x)$ is $d$-dimensional space-time white noise on $\mathbb{R}_{+} \times \mathbb{R}^{d}$.

$$
\mathcal{F}(\phi)=\int_{\mathbb{R}^{d}} \mathcal{A} \phi(x) W_{2}(d x)
$$

where $W_{2}(x)$ is a one-dimensional white noise on $\mathbb{R}^{d}$ independent of the white noise $W_{1}$. Observe that the stochastic differential equation (2.13) has two independent sources of randomness and thus we get the solution to (2.13) to be the sum of two independent stochastic integrals

$$
\begin{aligned}
Z(t, \phi)= & \sqrt{\rho_{0}} \int_{[0, t] \times \mathbb{R}^{d}} \nabla\left(T_{t-s} \phi(x)\right) \cdot \kappa W_{1}(d x d s) \\
& +\sqrt{v_{0}} \int_{0}^{t} \int_{\mathbb{R}^{d}} \mathcal{A} T_{t-s} \phi(x) W_{2}(d x) d s .
\end{aligned}
$$

Denote the two stochastic integrals in (2.16) as $Z_{1}(t, \phi)$ and $Z_{2}(t, \phi)$ so that

$$
Z(t, \phi)=\sqrt{\rho_{0}} Z_{1}(t, \phi)+\sqrt{v_{0}} Z_{2}(t, \phi) .
$$

The first term $\sqrt{\rho_{0}} Z_{1}(t, \phi)$ represents space-time noise created by fluctuations in the random walks. The second term $\sqrt{v_{0}} Z_{2}(t, \phi)$ indicates the fluctuations in the initial configuration that get propagated as the system evolves. In the absence of fluctuations in the initial configuration, i.e. if we take the initial configuration to be deterministic 
$\left(v_{0}=0\right)$, the second source of randomness in the spde $(2.13), \sqrt{v_{0}} \mathcal{F}(\cdot) d t$, disappears. The spde (2.13) in this case then reduces to

$$
d Z(t, \cdot)=\sqrt{\rho_{0}} d W_{t}^{\cdot}+\mathcal{A} Z(t, \cdot) d t
$$

where the solution

$$
Z(t, \phi)=\sqrt{\rho_{0}} \int_{[0, t] \times \mathbb{R}^{d}} \nabla\left(T_{t-s} \phi(x)\right) \cdot \kappa W_{1}(d x d s)
$$

is a generalized Ornstein-Uhlenbeck process [4].

For $t, s \in[0, T]$ and $\phi, \psi \in \mathcal{S}\left(\mathbb{R}^{d}\right)$,

$$
\operatorname{Cov}(Z(t, \phi), Z(s, \psi))=\rho_{0} \operatorname{Cov}\left(Z_{1}(t, \phi), Z_{1}(s, \psi)\right)+v_{0} \operatorname{Cov}\left(Z_{2}(t, \phi), Z_{2}(s, \psi)\right) \text {. }
$$

Elementary calculations show that

$$
\operatorname{Cov}\left(Z_{1}(t, \phi), Z_{1}(s, \psi)\right)=\sigma_{1}((s, \phi),(t, \psi))
$$

and

$$
\operatorname{Cov}\left(Z_{2}(t, \phi), Z_{2}(s, \psi)\right)=\sigma_{2}((s, \phi),(t, \psi)) .
$$

We can conclude that the solution of the spde and the limiting current process in Theorem 2.1 are equal in distribution as they are both mean zero, distribution-valued Gaussian processes with the same covariance.

\section{Convergence of Finite-Dimensional Distributions}

We begin the proof of Theorems 2.1 and 2.2 with showing convergence of finitedimensional distributions.

Note Henceforth, in this paper, ' $c$ ' will denote constants that change from line to line in calculations.

Fix $N \in \mathbb{N}$ and choose $\left(t_{i}, \phi_{i}\right) \in[0, T] \times \mathcal{S}\left(\mathbb{R}^{d}\right)$ for $i=1, \ldots, N$ such that $\left(t_{i}, \phi_{i}\right) \neq\left(t_{j}, \phi_{j}\right)$ for $i \neq j$. Let $\left(\theta_{1}, \ldots, \theta_{N}\right) \in \mathbb{R}^{N}$ be an arbitrary vector.

Lemma 3.1 As $n \rightarrow \infty, \sum_{i=1}^{N} \theta_{i} \xi_{n}\left(t_{i}, \phi_{i}\right)$ converges to a mean zero Gaussian random variable with variance

$$
\sigma^{2}=\sum_{i, j=1}^{d} \theta_{i} \theta_{j}\left(\rho_{0} \sigma_{1}\left(\left(t_{i}, \phi_{i}\right),\left(t_{j}, \phi_{j}\right)\right)+v_{0} \sigma_{2}\left(\left(t_{i}, \phi_{i}\right),\left(t_{j}, \phi_{j}\right)\right)\right) .
$$

Proof Define

$$
U_{m}(t, \phi):=n^{-d / 4} \sum_{j=1}^{\eta_{0}(m)}\left[\phi\left(\frac{X_{m, j}(n t)-[n \vec{v} t]}{\sqrt{n}}\right)-\phi\left(\frac{m}{\sqrt{n}}\right)\right] .
$$


Let $W_{m}=\sum_{i=1}^{N} \theta_{i} U_{m}\left(t_{i}, \phi_{i}\right)$. Denote $\bar{U}_{m}(t, \phi)=U_{m}(t, \phi)-E U_{m}(t, \phi)$ and $\bar{W}_{m}=$ $W_{m}-E W_{m}$. Choose $r(n)$ so that $r(n) \rightarrow \infty$ slowly enough that

$$
(r(n))^{d} \cdot E\left[\eta_{0}(x)^{2} 1\left\{\eta_{0}(x) \geq n^{d / 8}\right\}\right] \rightarrow 0 \quad \text { as } n \rightarrow \infty .
$$

Since $\left\{\eta_{0}(m): m \in \mathbb{Z}^{d}\right\}$ are i.i.d., a simple calculation shows that $E \eta_{t}(m)=\rho_{0}$, $\forall t>0, m \in \mathbb{Z}^{d}$ and hence $E \xi_{n}(t, \phi)=0$. Rewrite

$$
\sum_{i=1}^{N} \theta_{i} \xi_{n}\left(t_{i}, \phi_{i}\right)=\sum_{i=1}^{N} \theta_{i} \bar{\xi}_{n}\left(t_{i}, \phi_{i}\right)=\sum_{m \in \mathbb{Z}^{d}} \bar{W}_{m} .
$$

This sum can be split into the following two sums:

$$
\sum_{i=1}^{N} \theta_{i} \xi_{n}\left(t_{i}, \phi_{i}\right)=\sum_{|m| \leq r(n) \sqrt{n}} \bar{W}_{m}+\sum_{|m|>r(n) \sqrt{n}} \bar{W}_{m} .
$$

We can now apply the Lindeberg-Feller theorem to the sum $\sum_{|m| \leq r(n) \sqrt{n}} \bar{W}_{m}$ and show that it converges in distribution to the mean zero Gaussian random variable with variance indicated in Lemma 3.1. The proof follows in the same vein as the proof of Lemma 2 in [7] and we skip it.

We next show that the second sum in (3.2) converges to 0 in $L^{2}$ as $n \rightarrow \infty$. The proof of this differs from the analogous step in [7] and so we give the proof in detail. In [7] we use large deviations to control contributions to the current from distant particles. This is not enough in the present situation and we need to appeal to the rapidly decreasing property of Schwartz functions to bound these contributions.

By the Schwartz inequality,

$$
E\left(\sum_{|m|>r(n) \sqrt{n}} \bar{W}_{m}\right)^{2} \leq\|\theta\|^{2} \sum_{i=1}^{N} \sum_{|m|>r(n) \sqrt{n}} E \bar{U}_{m}\left(t_{i}, \phi_{i}\right)^{2}
$$

where $\|\theta\|^{2}=\sum_{i=1}^{N} \theta_{i}^{2}$. It suffices to show that for a fixed $t>0$ and $\phi \in \mathcal{S}\left(\mathbb{R}^{d}\right)$,

$$
\sum_{|m|>r(n) \sqrt{n}} E \bar{U}_{m}(t, \phi)^{2} \rightarrow 0
$$

as $n \rightarrow \infty$.

$$
\begin{aligned}
& \sum_{|m|>r(n) \sqrt{n}} E \bar{U}_{m}(t, \phi)^{2} \\
& \leq c n^{-d / 2} \sum_{|m|>r(n) \sqrt{n}} E\left[\sum_{j=1}^{\eta_{0}(m)} \phi\left(\frac{X_{m, j}(n t)-[n \vec{v} t]}{\sqrt{n}}\right)\right. \\
& \left.-\rho_{0} E \phi\left(\frac{X_{m, 1}(n t)-[n \vec{v} t]}{\sqrt{n}}\right)\right]^{2} \\
& +c n^{-d / 2} \sum_{|m|>r(n) \sqrt{n}} \phi^{2}\left(\frac{m}{\sqrt{n}}\right)
\end{aligned}
$$


since $(a+b)^{2} \leq 2 a^{2}+2 b^{2}$ and $\eta_{0}(\cdot)$ have bounded moments by Assumption 2.1

$$
\leq c n^{-d / 2}\left[\sum_{|m|>r(n) \sqrt{n}} E \phi^{2}\left(\frac{X_{m, 1}(n t)-[n \vec{v} t]}{\sqrt{n}}\right)+\sum_{|m|>r(n) \sqrt{n}} \phi^{2}\left(\frac{m}{\sqrt{n}}\right)\right]
$$

since $\eta_{0}(\cdot)$ have bounded second moments and are independent of the random walks

$$
\begin{aligned}
= & c n^{-d / 2} \sum_{|m|>r(n) \sqrt{n}}\left[E \left(\phi^{2}\left(\frac{X_{m, 1}(n t)-[n \vec{v} t]}{\sqrt{n}}\right)\right.\right. \\
& \left.\times \mathbf{1}\left\{X_{m, 1}(n t) \in B_{M(n) \sqrt{n}}+[n \vec{v} t]\right\}\right) \\
& +E\left(\phi^{2}\left(\frac{X_{m, 1}(n t)-[n \vec{v} t]}{\sqrt{n}}\right) \mathbf{1}\left\{X_{m, 1}(n t) \notin B_{M(n) \sqrt{n}}+[n \vec{v} t]\right\}\right) \\
& \left.+\phi^{2}\left(\frac{m}{\sqrt{n}}\right)\right]
\end{aligned}
$$

where $M(n)$ is chosen so that $M(n) \rightarrow \infty$ as $n \rightarrow \infty$ and $M(n)=o(r(n))$.

$$
=c n^{-d / 2}\left(I_{1}+I_{2}+I_{3}\right)
$$

where $I_{1}, I_{2}, I_{3}$ denote the sums of the three terms resp. in the expression above. We show below that the scaled limits of each of these sums goes to 0 as $n \rightarrow \infty$.

$$
n^{-d / 2} I_{1} \leq c n^{-d / 2} \sum_{|m|>r(n) \sqrt{n}} P\left(X(n t) \in B_{M(n) \sqrt{n}}+[n \vec{v} t]-m\right)
$$

since $\phi$ is bounded

$$
\begin{aligned}
& =c n^{-d / 2} \sum_{i \in B_{M(n) \sqrt{n}}} P\left(X(n t)-[n \vec{v} t] \notin B_{r(n) \sqrt{n}}+i\right) \\
& \leq c n^{-d / 2} \sum_{i \in B_{M(n) \sqrt{n}}} P\left(X(n t)-[n \vec{v} t] \notin B_{\frac{1}{2} r(n) \sqrt{n}}\right)
\end{aligned}
$$

as $\frac{1}{2} r(n) \sqrt{n} \leq r(n) \sqrt{n}-M(n) \sqrt{n}($ recall that $M(n)=o(r(n))$

$$
\begin{aligned}
& =c n^{-d / 2} \sum_{i \in B_{M(n) \sqrt{n}}} P\left(|X(n t)-[n \vec{v} t]|>\frac{1}{2} r(n) \sqrt{n}\right) \\
& \leq c n^{-d / 2} M(n)^{d} n^{d / 2} \frac{E|X(n t)-[n \vec{v} t]|^{r}}{(r(n) \sqrt{n})^{r}}
\end{aligned}
$$


by the Markov inequality, $r \geq 0$

$$
=c n^{r / 2} n^{-r / 2} M(n)^{d}(r(n))^{-r}
$$

(as $E|X(n t)-[n \vec{v} t]|^{r}$ is $\left.\mathrm{O}\left(n^{r / 2}\right)\right)$

$$
\rightarrow 0
$$

as $n \rightarrow \infty$ by taking $r>d$.

The following requires the rapidly decreasing property of Schwartz functions.

$$
\begin{aligned}
n^{-d / 2} I_{2} \leq & n^{-d / 2} \sum_{m \in B_{r(n) \sqrt{n}}^{c}} \sum_{L \geq M(n)} E\left(\phi^{2}\left(\frac{X_{m, 1}(n t)-[n \vec{v} t]}{\sqrt{n}}\right)\right. \\
& \times \mathbf{1}\left\{X_{m, 1}(n t) \in B_{L \sqrt{n}}^{c} \cap B_{(L+1) \sqrt{n}+[n \vec{v} t]\})}^{\leq}\right. \\
& n^{-d / 2} \sum_{m \in B_{r(n) \sqrt{n}}^{c}} \sum_{L \geq M(n)} \frac{c_{j}}{L^{2 j}} P\left(X(n t)-[n \vec{v} t] \in B_{L \sqrt{n}}^{c} \cap B_{(L+1) \sqrt{n}}-m\right)
\end{aligned}
$$

since $\phi \in \mathcal{S}\left(\mathbb{R}^{d}\right),|\phi(x)| \leq c_{j} x^{-j}$ for any $j \in \mathbb{N}$

$$
\begin{aligned}
& \leq n^{-d / 2} \sum_{m \in B_{r(n) \sqrt{n}}^{c}} \sum_{L \geq M(n)} \sum_{i \in B_{L \sqrt{n}}^{c} \cap B_{(L+1) \sqrt{n}}} \frac{c_{j}}{L^{2 j}} P(X(n t)-[n \vec{v} t]=i-m) \\
& \leq n^{-d / 2} \sum_{L \geq M(n)} \frac{c_{j}}{L^{2 j}}\left|B_{L \sqrt{n}}^{c} \cap B_{(L+1) \sqrt{n}}\right|
\end{aligned}
$$

by summing over $m$ first and then $i$

$$
\leq n^{-d / 2} \sum_{L \geq M(n)} \frac{c_{j}}{L^{2 j}}(L \sqrt{n})^{d-1} \sqrt{n}=\sum_{L \geq M(n)} \frac{c_{j}}{L^{2 j-d+1}} \rightarrow 0
$$

as $n \rightarrow \infty$ by choosing $2 j>d$ and since $M(n) \rightarrow \infty$ as $n \rightarrow \infty$. And finally,

$$
n^{-d / 2} I_{3} \leq c \int_{|x|>r(n)} \phi^{2}(x) d x \rightarrow 0
$$

as $n \rightarrow \infty$ by a Riemann sum argument and since $r(n) \rightarrow \infty$ as $n \rightarrow \infty$.

Thus the right hand side of (3.3) goes to 0 as $n \rightarrow \infty$ and since

$$
E\left(\sum_{|m|>r(n) \sqrt{n}} \bar{W}_{m}\right)^{2} \leq\|\theta\|^{2} \sum_{i=1}^{N} \sum_{|m|>r(n) \sqrt{n}} E \bar{U}_{m}\left(t_{i}, \phi_{i}\right)^{2},
$$

we conclude that the second sum in (3.2) goes to 0 in $L^{2}$. This proves Lemma 3.1. 


\section{Tightness and Completion of the Proof}

Choose $\alpha>0$ and

$$
\beta \geq d / 4+d \alpha+1
$$

Fix $\phi \in \mathcal{S}\left(\mathbb{R}^{d}\right)$. We prove

Proposition 4.1 $\xi_{n}(\cdot, \phi)$ is tight in $D([0, T], \mathbb{R})$.

To prove Proposition 4.1 we use the tightness criteria in [2]. In Sects. 4.1 and 4.2 below, we check the two tightness conditions of Proposition 5.7 in [2] for the sequence of processes $\left\{\xi_{n}(\cdot, \phi)\right\}$. The first condition is a moment bound condition and the second involves the modulus of continuity.

\subsection{Verifying the First Tightness Condition}

Let $s, t \in[0, T]$ and without loss of generality we assume $t>s$ below. Let $\bar{\xi}$ denote the centered current process. Choose an integer

$$
r>2 \beta>2 \text {. }
$$

We show that

Lemma 4.1 When $(t-s) \geq n^{-\beta}$,

$$
E\left|\bar{\xi}_{n}(t, \phi)-\bar{\xi}_{n}(s, \phi)\right|^{2 r} \leq C_{r}(t-s)^{\sigma}
$$

where $\sigma>1$, and $C_{r}$ is a constant depending only on $r$.

Proof Let

$$
A_{m}:=\sum_{j=1}^{\eta_{0}(m)}\left(\phi\left(\frac{X_{m, j}(n t)-[n \vec{v} t]}{\sqrt{n}}\right)-\phi\left(\frac{X_{m, j}(n s)-[n \vec{v} s]}{\sqrt{n}}\right)\right) .
$$

We can write

$$
\xi_{n}(t, \phi)-\xi_{n}(s, \phi)=n^{-d / 4} \sum_{m \in \mathbb{Z}^{d}} A_{m}
$$

Then

$$
E\left|\bar{\xi}_{n}(t, \phi)-\bar{\xi}_{n}(s, \phi)\right|^{2 r}=n^{-r d / 2} E\left(\sum_{m \in \mathbb{Z}^{d}} \bar{A}_{m}\right)^{2 r}
$$

where $\bar{A}_{m}=A_{m}-E A_{m}$.

To appropriately bound (4.4), we first find the following moment bound. 
Lemma 4.2 For any integer $1 \leq k \leq 2 r$, there exists a constant $C$ that depends on $r$, such that

$$
\sum_{m \in \mathbb{Z}^{d}} E\left|\bar{A}_{m}\right|^{k} \leq C\left(n^{-1 / 2}+\sqrt{t-s}\right) n^{d / 2}
$$

\section{Proof}

$$
\sum_{m \in \mathbb{Z}^{d}} E\left|\bar{A}_{m}\right|^{k} \leq 2^{k} \sum_{m \in \mathbb{Z}^{d}}\left[E\left|\bar{A}_{m}\right|^{k}\right]
$$

by applying $(a+b)^{k} \leq 2^{k}\left(|a|^{k}+|b|^{k}\right)$ and then Hölder's inequality

$$
\begin{aligned}
\leq & 2^{k} \sum_{m \in \mathbb{Z}^{d}}\left[E\left(\eta_{0}(m)\right)^{k} E \mid \phi\left(\frac{X_{m, 1}(n t)-[n \vec{v} t]}{\sqrt{n}}\right)\right. \\
& \left.-\left.\phi\left(\frac{X_{m, 1}(n s)-[n \vec{v} s]}{\sqrt{n}}\right)\right|^{k}\right]
\end{aligned}
$$

by Jensen's inequality and independence of $\eta_{0}(\cdot)$ from the random walks

$$
\leq c 2^{2 r}\left[\sum_{m \in \mathbb{Z}^{d}} E\left|\phi\left(\frac{X_{m, 1}(n t)-[n \vec{v} t]}{\sqrt{n}}\right)-\phi\left(\frac{X_{m, 1}(n s)-[n \vec{v} s]}{\sqrt{n}}\right)\right|^{k}\right]
$$

since $\eta_{0}(m)$ has bounded moments.

Define

$$
D \phi(x):=\left(\partial_{1} \phi(x), \ldots, \partial_{d} \phi(x)\right),
$$

where $\partial_{i}$ denotes the partial derivative with respect to the $i$ th co-ordinate of $x$. Let $\psi(x):=(1+|x|)^{-N}$ for some positive integer $N$.

$$
\begin{gathered}
\sum_{m \in \mathbb{Z}^{d}} E\left|\phi\left(\frac{X_{m, 1}(n t)-[n \vec{v} t]}{\sqrt{n}}\right)-\phi\left(\frac{X_{m, 1}(n s)-[n \vec{v} s]}{\sqrt{n}}\right)\right|^{k} \\
\leq c_{N} \sum_{m \in \mathbb{Z}^{d}}\left[E \left[\left(\psi\left(\frac{X_{m, 1}(n s)-[n \vec{v} s]}{\sqrt{n}}\right)\right)^{k} \mid \frac{X_{m, 1}(n t)-[n \vec{v} t]}{\sqrt{n}}\right.\right. \\
\quad-\left.\frac{X_{m, 1}(n s)-[n \vec{v} s]}{\sqrt{n}}\right|^{k} \\
\left.\quad \times \mathbf{1}\left\{\left|\frac{X_{m, 1}(n t)-[n \vec{v} t]}{\sqrt{n}}\right| \geq\left|\frac{X_{m, 1}(n s)-[n \vec{v} s]}{\sqrt{n}}\right|\right\}\right]
\end{gathered}
$$




$$
\begin{aligned}
& +E\left[\left(\psi\left(\frac{X_{m, 1}(n t)-[n \vec{v} t]}{\sqrt{n}}\right)\right)^{k}\left|\frac{X_{m, 1}(n t)-[n \vec{v} t]}{\sqrt{n}}-\frac{X_{m, 1}(n s)-[n \vec{v} s]}{\sqrt{n}}\right|^{k}\right. \\
& \left.\left.\times \mathbf{1}\left\{\left|\frac{X_{m, 1}(n s)-[n \vec{v} s]}{\sqrt{n}}\right|>\left|\frac{X_{m, 1}(n t)-[n \vec{v} t]}{\sqrt{n}}\right|\right\}\right]\right]
\end{aligned}
$$

by applying the mean value theorem and $|D \phi(x)| \leq c_{N} \psi(x)$.

We will treat one of the terms in the above inequality, the other being similar.

$$
\begin{aligned}
c_{N} \sum_{m \in \mathbb{Z}^{d}} & {\left[E \left[\left(\psi\left(\frac{X_{m, 1}(n s)-[n \vec{v} s]}{\sqrt{n}}\right)\right)^{k}\left|\frac{X_{m, 1}(n t)-[n \vec{v} t]}{\sqrt{n}}-\frac{X_{m, 1}(n s)-[n \vec{v} s]}{\sqrt{n}}\right|^{k}\right.\right.} \\
& \left.\times \mathbf{1}\left\{\left|\frac{X_{m, 1}(n t)-[n \vec{v} t]}{\sqrt{n}}\right| \geq\left|\frac{X_{m, 1}(n s)-[n \vec{v} s]}{\sqrt{n}}\right|\right\}\right] \\
\leq & c_{N} \sum_{m \in \mathbb{Z}^{d}} \sum_{L \geq 0} E\left[\frac{1}{(1+L)^{k N}}\left|\frac{X_{m, 1}(n t)-[n \vec{v} t]}{\sqrt{n}}-\frac{X_{m, 1}(n s)-[n \vec{v} s]}{\sqrt{n}}\right|^{k}\right. \\
& \left.\times \mathbf{1}\left\{\frac{X_{m, 1}(n s)-[n \vec{v} s]}{\sqrt{n}} \in B_{L}^{c} \cap B_{(L+1)}\right\}\right] \\
\leq & c_{N} \sum_{m \in \mathbb{Z}^{d}} \sum_{L \geq 0} \frac{1}{(1+L)^{k N}} E\left[\left|\frac{X(n t)-X(n s)-[n \vec{v} t]+[n \vec{v} s]}{\sqrt{n}}\right|^{k}\right. \\
& \times \mathbf{1}\left\{X(n s) \in B_{L \sqrt{n}}^{c} \cap B_{(L+1) \sqrt{n}+[n \vec{v} s]-m\}]}\right. \\
\leq & c_{N} \sum_{L \geq 0} \frac{1}{(1+L)^{k N}((L+1) \sqrt{n})^{d-1} \sqrt{n} E\left|\frac{X(n t)-X(n s)-[n \vec{v} t]+[n \vec{v} s]}{\sqrt{n}}\right|^{k}}
\end{aligned}
$$

by summing over $m$

$$
\leq c_{N}\left[\sum_{L \geq 0}(1+L)^{-k N+d-1}\right] n^{d / 2}\left[(t-s)^{k / 2}+n^{-k / 2}\right]
$$

using central limit theorem

$$
\leq c n^{d / 2}\left[\sqrt{t-s}+n^{-1 / 2}\right]
$$

by choosing $N$ large enough so that the sum in parentheses is finite and by bounding $(t-s)^{k / 2} \leq(2 T)^{\frac{k-1}{2}} \sqrt{t-s}$ and $n^{-k / 2} \leq n^{-1 / 2}$. 
Lemma 4.3 There exists a constant $C$ depending on $r$ such that

$$
E\left(\sum_{m \in \mathbb{Z}^{d}} \bar{A}_{m}\right)^{2 r} \leq C\left\{1+\left(n^{-r / 2}+(t-s)^{r / 2}\right) n^{r d / 2}\right\}
$$

The proof of the above lemma uses Lemma 4.2 and is along the same lines as Lemma 8 in [7]. (In fact Lemma 8 in [7] is a special case where $r$ is 6.) We omit the proof. The reader is referred to Lemma 8 in [7] for the idea of the proof.

By (4.4) and the above lemma we get, when $|t-s| \geq n^{-\beta}$,

$$
\begin{aligned}
E\left|\bar{\xi}_{n}(t, \phi)-\bar{\xi}_{n}(s, \phi)\right|^{2 r} & \leq c\left[n^{-r d / 2}+n^{-r / 2}+(t-s)^{r / 2}\right] \\
& \leq c\left[\left(n^{-\beta}\right)^{\frac{r d}{2 \beta}}+\left(n^{-\beta}\right)^{\frac{r}{2 \beta}}+(t-s)^{r / 2}\right] \\
& \leq c(t-s)^{\sigma}
\end{aligned}
$$

where $\sigma>1$ by our choice of $r>2 \beta>2$ in (4.2) and where $c$ is a constant depending on $r$.

\subsection{Verifying the Second Tightness Condition}

The second tightness condition involves proving that

$$
\lim _{n \rightarrow \infty} P\left(w_{\xi_{n}}\left(n^{-\beta}\right)>\epsilon\right)=0
$$

where

$$
w_{\xi_{n}}\left(n^{-\beta}\right):=\sup _{|t-s|<n^{-\beta}}\left|\xi_{n}(t, \phi)-\xi_{n}(s, \phi)\right|
$$

is the modulus of continuity.

Recall the definition of the step function [-] in (2.1). For each $n$, we will divide $[0, T]$ into subintervals such that $[n \vec{v} t]$ is constant in each subinterval and each subinterval has length less than $n^{-\beta}$. We construct a family $\mathcal{I}_{n}$ of left-closed right-open subintervals by following the steps below.

Step 1: Set $a=0$.

Step 2: Let $b_{1}$ be the largest real number greater than $a$ such that $[n \vec{v} t]$ is constant as $t$ varies over interval $\left[a, b_{1}\right)$.

Step 3: Set $b=\min \left\{b_{1}, a+n^{-\beta}\right\}$.

Step 4: Include $[a, b)$ in $\mathcal{I}_{n}$.

Step 5: If $b>T$ then stop, otherwise set $a=b$ and repeat from Step 2.

Clearly $\mathcal{I}_{n}$ forms a minimal covering of $[0, T]$ that satisfies the following two conditions:

(1) $\forall[a, b) \in \mathcal{I}_{n},|b-a|<n^{-\beta}$ and $[n \vec{v} t]$ is constant in $[a, b)$,

(2) the subintervals in $\mathcal{I}_{n}$ are disjoint. 
A little thought gives us

$$
\left|\mathcal{I}_{n}\right| \leq d\left[T n^{\beta}+1\right] \text { for large } n
$$

(i.e. number of such subintervals is at most $d\left[T n^{\beta}+1\right]$ where $d$ is the dimension).

To verify the second tightness condition, it is sufficient to show

Lemma 4.4 For any $0<T<\infty$ and $\epsilon>0$,

$$
\lim _{n \rightarrow \infty} \sum_{[a, b) \in \mathcal{I}_{n}} P\left(\sup _{t \in[a, b)}\left|\xi_{n}(t, \phi)-\xi_{n}(a, \phi)\right|>\epsilon\right)=0 .
$$

The reason that Lemma 4.4 is sufficient to prove (4.7) is the following. For any $0 \leq$ $s \leq t \leq T$ with $t-s<n^{-\beta}$, there exist $\left[a_{1}, b_{1}\right),\left[a_{2}, b_{2}\right) \in \mathcal{I}_{n}$ such that $s \in\left[a_{1}, b_{1}\right)$ and $t \in\left[a_{2}, b_{2}\right)\left(\left[a_{1}, b_{1}\right)\right.$ may be equal to $\left.\left[a_{2}, b_{2}\right)\right)$. By the triangle inequality we get

$$
\begin{aligned}
& \left|\xi_{n}(t, \phi)-\xi_{n}(s, \phi)\right| \\
& \leq\left|\xi_{n}(t, \phi)-\xi_{n}\left(a_{2}, \phi\right)\right|+\left|\xi_{n}\left(a_{2}, \phi\right)-\xi_{n}\left(a_{2}+n^{-\beta}, \phi\right)\right| \\
& \quad+\left|\xi_{n}\left(a_{2}+n^{-\beta}, \phi\right)-\xi_{n}\left(a_{1}, \phi\right)\right|+\left|\xi_{n}\left(a_{1}, \phi\right)-\xi_{n}(s, \phi)\right|
\end{aligned}
$$

By applying Lemma 4.1 and $\left|\mathcal{I}_{n}\right| \leq d\left[T n^{\beta}+1\right]$, we get

$$
P\left(w_{\xi_{n}}\left(n^{-\beta}\right)>\epsilon\right) \leq 2 \sum_{[a, b) \in \mathcal{I}_{n}} P\left(\sup _{t \in[a, b)}\left|\xi_{n}(t, \phi)-\xi_{n}(a, \phi)\right|>\epsilon / 4\right)+c n^{\beta(1-\sigma)}
$$

As $n \rightarrow \infty$, the second term in the above inequality goes to 0 as $\sigma>1$.

Proof of Lemma 4.4 Let

$$
(\dagger):=\sum_{[a, b) \in \mathcal{I}_{n}} P\left(\sup _{t \in[a, b)}\left|\xi_{n}(t, \phi)-\xi_{n}(a, \phi)\right|>\epsilon\right) .
$$

We want to show that

$$
\lim _{n \rightarrow \infty}(\dagger)=0 \text {. }
$$

We start by giving a general idea of the proof. The current process is written as the sum of two parts: contributions from particles starting inside the box $B_{n^{1 / 2+\alpha}}$ (recall $\alpha>0$ from beginning of this section) and contributions from particles starting outside the box $B_{n^{1 / 2+\alpha}}$. In the first case we bound the current by the number of jumps executed by particles initially within the box $B_{n^{1 / 2+\alpha}}$. In the second case we conclude that particles starting outside the box $B_{n^{1 / 2+\alpha}}$ can either travel a distance of order greater than $\sqrt{n}$ toward the origin, which would be a large deviation, or they would remain sufficiently far from the origin, in which case the value of $\phi$ at that distance from the origin would be small.

Choose $\gamma$ such that

$$
0<\gamma<\alpha
$$


We first find a bound on the expected number of particles starting outside the box $B_{n^{1 / 2+\alpha}}$, which enter the box $B_{n^{1 / 2+\gamma}}$ at some point in time interval $[0, n T]$.

\section{Lemma 4.5 Define}

$$
\begin{gathered}
N_{1}:=\sum_{|m|>n^{1 / 2+\alpha}} \sum_{j=1}^{\eta_{0}(m)} \mathbf{1}\left\{X_{m, j}(n t) \in B_{n^{1 / 2+\gamma}}+[n \vec{v} t]\right. \\
\text { for some } 0 \leq t \leq T\} .
\end{gathered}
$$

Let $l \geq 1$. Then there exists a constant $c$ independent of $n$ such that $E N_{1} \leq$ $c n^{d / 2-l \alpha+d \gamma}$.

Proof Recall that $X(n t)$ denotes a random walk starting at the origin, with the same distribution as the evolution of the particles. Let $\bar{X}(n t):=X(n t)-[n \vec{v} t]$.

$$
E N_{1}=\rho_{0} \sum_{|m|>n^{1 / 2+\alpha}} P\left(\bar{X}(n t) \in B_{n^{1 / 2+\gamma}}-m \text { for some } t \in[0, T]\right)
$$

since $\eta_{0}$ is independent of the random walks

$$
\begin{aligned}
& =\rho_{0} \sum_{i \in B_{n^{1 / 2+\gamma}}} P\left(\bar{X}(n t) \notin B_{n^{1 / 2+\alpha}}+i \text { for some } t \in[0, T]\right) \\
& \leq \rho_{0} \sum_{i \in B_{n^{1 / 2}+\gamma}} P\left(\bar{X}(n t) \notin B_{\frac{1}{2} n^{1 / 2+\alpha}} \text { for some } t \in[0, T]\right)
\end{aligned}
$$

for large enough $n, \frac{1}{2} n^{1 / 2+\alpha} \leq n^{1 / 2+\alpha}-n^{1 / 2+\gamma}$, as $\gamma<\alpha$

$$
\begin{aligned}
& =\rho_{0} \sum_{i \in B_{n^{1 / 2}+\gamma}} P\left(\sup _{0 \leq t \leq T}|\bar{X}(n t)|>\frac{1}{2} n^{1 / 2+\alpha}\right) \\
& \leq c n^{d / 2}\left(n^{\gamma}\right)^{d} \frac{E|\bar{X}(n T)|^{l}}{\left(\frac{1}{2} n^{1 / 2+\alpha}\right)^{l}}
\end{aligned}
$$

by Doob's inequality for any $l \geq 1$

$$
\leq c n^{d / 2}\left(n^{\gamma}\right)^{d} n^{l / 2} n^{-l \alpha-l / 2}
$$

as $E \mid \bar{X}(n T))\left.\right|^{l}$ is $\mathrm{O}\left(n^{l / 2}\right)$

$$
\leq c n^{d / 2-l \alpha+d \gamma}
$$


Let $[a, b) \in \mathcal{I}_{n}$. Define

$$
h_{m, j}^{[a, b)}(t):=\phi\left(\frac{X_{m, j}(n t)-[n \vec{v} t]}{\sqrt{n}}\right)-\phi\left(\frac{X_{m, j}(n a)-[n \vec{v} a]}{\sqrt{n}}\right) .
$$

We can rewrite $(\dagger)$ in (4.11) as

$$
\begin{aligned}
(\dagger)= & \sum_{[a, b) \in \mathcal{I}_{n}} P\left(\sup _{t \in[a, b)}\left|\sum_{m \in B_{n^{1 / 2+\alpha}}} \sum_{j=1}^{\eta_{0}(m)} h_{m, j}^{[a, b)}(t)\right|>\frac{n^{d / 4} \epsilon}{2}\right) \\
& +\sum_{[a, b) \in \mathcal{I}_{n}} P\left(\sup _{t \in[a, b)}\left|\sum_{m \in B_{n}^{c} 1 / 2+\alpha} \sum_{j=1}^{\eta_{0}(m)} h_{m, j}^{[a, b)}(t)\right|>\frac{n^{d / 4} \epsilon}{2}\right)
\end{aligned}
$$

The two sums reflect the split in contributions to the current process from particles starting within $B_{n^{1 / 2+\alpha}}$ in (4.14a), versus particles starting outside $B_{n^{1 / 2+\alpha}}$ in (4.14b).

We first show that the second term (4.14b) goes to 0 as $n \rightarrow \infty$. To do this we split (4.14b) into two sums, the first containing contributions from particles that enter $B_{n^{1 / 2}+\gamma}$ at some time in $[0, n T]$ and the second containing contributions from particles that never enter $B_{n^{1 / 2+\gamma}}$.

$$
\begin{aligned}
& \sum_{[a, b) \in \mathcal{I}_{n}} P\left(\sup _{t \in[a, b)}\left|\sum_{m \in B_{n^{1 / 2+\alpha}}} \sum_{j=1}^{\eta_{0}(m)} h_{m, j}^{[a, b)}(t)\right|>\frac{n^{d / 4} \epsilon}{2}\right) \\
& \leq \sum_{[a, b) \in \mathcal{I}_{n}} P\left(\sum _ { m \in B _ { n ^ { 1 / 2 + \alpha } } ^ { c } } \sum _ { j = 1 } ^ { \eta _ { 0 } ( m ) } c \mathbf { 1 } \left\{X_{m, j}(n t) \in B_{n^{1 / 2+\gamma}}+[n \vec{v} t]\right.\right. \\
& \left.\quad \text { for some } t \in[0, T]\}>\frac{n^{d / 4} \epsilon}{4}\right) \\
& +\sum_{[a, b) \in \mathcal{I}_{n}} P\left(\sup _{t \in[a, b)} \sum_{m \in B_{n}^{c}{ }_{n}^{1 / 2+\alpha}} \sum_{j=1}^{\eta_{0}(m)}\left|h_{m, j}^{[a, b)}(t)\right|\right. \\
& \left.\quad \times \mathbb{1}\left\{X_{m, j}(n t) \in B_{n^{1 / 2}+\gamma}^{c}+[n \vec{v} t], \forall t \in[a, b)\right\}>\frac{n^{d / 4} \epsilon}{4}\right)
\end{aligned}
$$

To get (4.15a), we bounded $h_{m, j}^{[a, b)}$ by some constant $c$ times the indicator function, since $\phi$ is a bounded function.

Now using Lemma 4.5, we get

$$
\begin{aligned}
(4.15 \mathrm{a}) & \leq \sum_{[a, b) \in \mathcal{I}_{n}} P\left(c N_{1} \geq \frac{n^{d / 4} \epsilon}{2}\right) \\
& \leq c n^{\beta} n^{-d / 4} E N_{1}
\end{aligned}
$$


by Markov inequality and since $\left|\mathcal{I}_{n}\right| \leq d\left[T n^{\beta}+1\right]$

$$
\leq c n^{\beta} n^{-d / 4} n^{d / 2-l \alpha+d \gamma}
$$

by Lemma 4.5 . Choose ' $l$ ' large enough so that $\beta+d / 4-l \alpha+d \gamma<0$. Then the right hand side $\rightarrow 0$ as $n \rightarrow \infty$.

We use the property that Schwartz functions are rapidly decreasing to show (4.15b) goes to 0 .

$$
\begin{aligned}
(4.15 b) \leq & \sum_{[a, b) \in \mathcal{I}_{n}} P\left(\sum_{m \in B_{n^{c} / 2+\alpha}} \sum_{j=1}^{\eta_{0}(m)} \sum_{L \geq n^{\gamma}} c_{N}(1+L)^{-N}\right. \\
& \left.\times \mathbf{1}\left\{L \leq \inf _{t \in[a, b)}\left|\frac{X_{m, j}(n t)-[n \vec{v} t]}{\sqrt{n}}\right| \leq(L+1)\right\}>\frac{n^{d / 4} \epsilon}{4}\right)
\end{aligned}
$$

since $|\phi(x)| \leq c_{N}(1+|x|)^{-N}$

$$
\begin{aligned}
\leq & \frac{n^{-d / 4} 4}{\epsilon} \rho_{0} \sum_{[a, b) \in \mathcal{I}_{n}} \sum_{m \in B_{n^{1 / 2+\alpha}}^{c}} \sum_{L \geq n^{\gamma}} c_{N}(1+L)^{-N} \\
& \times P\left(\frac{X_{m, 1}(n t)-[n \vec{v} t]}{\sqrt{n}} \in B_{L}^{c} \cap B_{(L+1)} \text { for some } t \in[a, b)\right)
\end{aligned}
$$

by the Markov inequality and since $\eta_{0}$ is independent of the random walks

$$
\begin{aligned}
= & \frac{n^{-d / 4} 4}{\epsilon} \rho_{0} \sum_{[a, b) \in \mathcal{I}_{n}} \sum_{m \in B_{n^{1 / 2+\alpha}}} \sum_{L \geq n^{\gamma}} c_{N}(1+L)^{-N} \\
& \times P\left(X(n t)-[n \vec{v} t] \in B_{L \sqrt{n}}^{c} \cap B_{(L+1) \sqrt{n}-m \text { for some } t \in[a, b))}^{\leq}\right. \\
\leq & \frac{n^{-d / 4}}{\epsilon} c_{N} \sum_{[a, b) \in \mathcal{I}_{n}} \sum_{L \geq n^{\gamma}}(1+L)^{-N}(L \sqrt{n})^{d-1} \sqrt{n}
\end{aligned}
$$

by summing over $m$ and allowing $c_{N}$ to absorb all the other constants

$$
\leq c_{N} \frac{n^{d / 4+\beta}}{\epsilon} \sum_{L \geq n^{\gamma}} L^{-N+d-1}
$$

since $\left|\mathcal{I}_{n}\right| \leq d\left[T n^{\beta}+1\right]$

$$
\leq c_{N} n^{d / 4+\beta-\gamma(N-d)} \rightarrow 0
$$

by choosing $N$ large enough so that $d / 4+\beta-\gamma(N-d)<0$. 
We finally turn to (4.14a). To prove (4.14a) goes to 0 , we show that the number of particles initially inside $B_{n^{1 / 2+\alpha}}$ that jump during a time interval of length $n^{1-\beta}$, is stochastically smaller than $O\left(n^{d / 4}\right)$.

Recall, by definition of $\mathcal{I}_{n},[n \vec{v} t]$ is constant for $t \in[a, b) \in \mathcal{I}_{n}$. Therefore for $t \in$ $[a, b)$,

$$
\left|h_{m, j}^{[a, b)}(t)\right| \leq C \mathbf{1}\left\{G_{m, j}^{[a, b)}\right\}
$$

where $G_{m, j}^{[a, b)}:=\left\{X_{m, j}(n t)\right.$ jumps during time interval $\left.t \in[a, b)\right\}$ and $C=$ $2 \sup _{x \in \mathbb{R}^{d}} \phi(x)$. Define

$$
\Lambda_{n}^{[a, b)}:=\log E\left[\exp \left\{\sum_{m \in B_{n} 1 / 2+\alpha} \sum_{j=1}^{\eta_{0}(m)} \mathbf{1}\left\{G_{m, j}^{[a, b)}\right\}\right\}\right]
$$

and

$$
\tilde{\Lambda}_{n}^{[a, b)}:=\log E\left[\exp \left\{\mathbf{1}\left\{\mathbf{G}_{\mathbf{m}, \mathbf{1}}^{[\mathbf{a}, \mathbf{b})}\right\}\right\}\right] .
$$

Let $\Pi\left(n^{1-\beta}\right)$ be a Poisson $\left(n^{1-\beta}\right)$ random variable.

$$
\begin{aligned}
\tilde{\Lambda}_{n}^{[a, b)} & =\log \left\{1+(e-1) P\left(X_{m, 1}(n t) \text { jumps during time interval } t \in[a, b)\right)\right\} \\
& \leq \log \left\{1+(e-1) P\left(\Pi\left(n^{1-\beta}\right) \geq 1\right)\right\}
\end{aligned}
$$

since $b-a \leq n^{-\beta}$

$$
\begin{aligned}
& \leq(e-1) P\left(\Pi\left(n^{1-\beta}\right) \geq 1\right) \\
& =c n^{1-\beta}
\end{aligned}
$$

by the Markov inequality.

$$
\Lambda_{n}^{[a, b)}=\sum_{m \in B_{n} 1 / 2+\alpha} \log E \exp \left\{\eta_{0}(m) \tilde{\Lambda}_{n}^{[a, b)}\right\}
$$

by independence of $\eta_{0}$ from the random walks

$$
\leq \sum_{m \in B_{n} 1 / 2+\alpha} \log \left[1+\rho_{0} c n^{1-\beta}(e-1)+o\left(n^{1-\beta}\right)\right]
$$

by (4.16) and since $\eta_{0}(m)$ has exponential moments

$$
\leq \sum_{m \in B_{n} 1 / 2+\alpha} c n^{1-\beta} \leq c n^{d / 2+d \alpha+1-\beta}
$$


Putting all this together, we get

$$
(4.14 \mathrm{a}) \leq \sum_{[a, b) \in \mathcal{I}_{n}} P\left(\sum_{m \in B_{n^{1 / 2+\alpha}}} \sum_{j=1}^{\eta_{0}(m)} \mathbf{1}\left\{G_{m, j}^{[a, b)}\right\}>\frac{n^{d / 4} \epsilon}{2 C}\right)
$$

since $\left|h_{m, j}^{[a, b)}(t)\right| \leq C \mathbf{1}\left\{G_{m, j}^{[a, b)}\right\}$ for $t \in[a, b)$

$$
\leq \sum_{[a, b) \in \mathcal{I}_{n}} \exp \left\{\frac{-n^{d / 4} \epsilon}{2 C}\right\} E\left[\exp \left\{\sum_{m \in B_{n^{1 / 2+\alpha}}} \sum_{j=1}^{\eta_{0}(m)} \mathbf{1}\left\{G_{m, j}^{[a, b)}\right\}\right\}\right]
$$

by the Markov inequality

$$
\begin{aligned}
& \leq \sum_{[a, b) \in \mathcal{I}_{n}} \exp \left\{\frac{-n^{d / 4} \epsilon}{2 C}\right\} \exp \left\{\Lambda_{n}^{[a, b)}\right\} \\
& \leq d\left[T n^{\beta}+1\right] \exp \left\{-c n^{d / 4}\left(1-n^{d / 4+d \alpha+1-\beta}\right)\right\}
\end{aligned}
$$

by applying (4.17) and $\left|\mathcal{I}_{n}\right| \leq d\left[T n^{\beta}+1\right]$

$$
\rightarrow 0
$$

as $n \rightarrow \infty$, since $\beta \geq d / 4+d \alpha+1$ by definition (4.1).

This proves Lemma 4.4 and thus verifies the second tightness condition (4.7).

Proof of Proposition 4.1 Lemma 4.1 and (4.7) satisfy the tightness criteria in Proposition 5.7 of [2], thus we get $\xi_{n}(\cdot, \phi)$ is tight in $D([0, T], \mathbb{R})$.

Proof of Theorem 2.1 We invoke Theorem 4.1 in [9] which states that Proposition 4.1 is sufficient to prove that the sequence $\left\{\xi_{n}(\cdot, \cdot)\right\}$ is tight in $D\left([0, T], \mathcal{S}^{\prime}\left(\mathbb{R}^{d}\right)\right)$. This, together with Lemma 3.1 proves the theorem.

Proof of Theorem 2.2 The proof of this theorem follows almost verbatim from that of Theorem 2.1. A few places where the proof differs from that of Theorem 2.1 are highlighted below.

When proving tightness, the proof of Lemma 4.2 is different. From (4.5) onward in Lemma 4.2, the proof differs as follows.

$$
\begin{aligned}
\sum_{m \in \mathbb{Z}^{d}} E\left|\bar{A}_{m}\right|^{k} & \leq c 2^{2 r}\left[\sum_{m \in \mathbb{Z}^{d}} E\left|\phi\left(\frac{X_{m, 1}(n t)-[n \vec{v} t]}{\sqrt{n}}\right)-\phi\left(\frac{X_{m, 1}(n s)-[n \vec{v} s]}{\sqrt{n}}\right)\right|^{k}\right] \\
& \leq c 2^{2 r} \sum_{m \in \mathbb{Z}^{d}}\left[P\left(C_{m}\right)+P\left(D_{m}\right)\right]
\end{aligned}
$$


where $C_{m}=\left\{X_{m, 1}(n t) \in B_{M \sqrt{n}}+[n \vec{v} t], X_{m, 1}(n s) \notin B_{M \sqrt{n}}+[n \vec{v} s]\right\}$ and $D_{m}=$ $\left\{X_{m, 1}(n t) \notin B_{M \sqrt{n}}+[n \vec{v} t], X_{m, 1}(n s) \in B_{M \sqrt{n}}+[n \vec{v} s]\right\}$.

$$
\begin{aligned}
& \sum_{m \in \mathbb{Z}^{d}} P\left(C_{m}\right)=P\left(X(n t) \in B_{M \sqrt{n}}+[n \vec{v} t]-m, X(n s) \notin B_{M \sqrt{n}}+[n \vec{v} s]-m\right) \\
& =\sum_{m \in \mathbb{Z}^{d}} \sum_{j \notin B_{M \sqrt{n}}} P\left(X(n t)-X(n s) \in B_{M \sqrt{n}}+[n \vec{v} t]-[n \vec{v} s]-j\right. \\
& \mid X(n s)=j+[n \vec{v} s]-m) \times P(X(n s)=j+[n \vec{v} s]-m) \\
& =\sum_{j \notin B_{M \sqrt{n}}} P\left(X(n(t-s)) \in B_{M \sqrt{n}}+[n \vec{v} t]-[n \vec{v} s]-j\right) \\
& =\sum_{j \notin B_{M \sqrt{n}}} P\left(\bar{X}_{n}(t, s) \in B_{M \sqrt{n}}+j\right)
\end{aligned}
$$

where $\bar{X}_{n}(t, s)=X(n(t-s))-[n \vec{v} t]+[n \vec{v} s]$

$$
\begin{aligned}
= & \sum_{k \in \mathbb{Z}^{d}} P\left(\bar{X}_{n}(t, s)=k\right) \sum_{j \notin B_{M \sqrt{n}}} \mathbf{1}\left\{k \in B_{M \sqrt{n}}+j\right\} \\
\leq & \sum_{l=0}^{M \sqrt{n}} \sum_{k \in \mathbb{Z}^{d}:|k|=l} P\left(\bar{X}_{n}(t, s)=k\right) n^{\frac{d-1}{2}}|k| \\
& +\sum_{|k|>M \sqrt{n}} P\left(\bar{X}_{n}(t, s)=k\right) c n^{d / 2} \\
\leq & n^{\frac{d-1}{2}} E\left|\bar{X}_{n}(t, s)\right|+c n^{d / 2} P\left(\bar{X}_{n}(t, s)>M \sqrt{n}\right) \\
\leq & c\left[n^{\frac{d-1}{2}}(\sqrt{n(t-s)}+1)+n^{d / 2} \frac{E\left|\bar{X}_{n}(t, s)\right|^{2}}{M^{2} n}\right]
\end{aligned}
$$

since $E|X(n(t-s))-n(t-s) \vec{v}|$ is $O(\sqrt{n(t-s)})$

$$
\begin{aligned}
& \leq c\left[n^{\frac{d-1}{2}}(\sqrt{n(t-s)}+1)+n^{d / 2} \frac{n(t-s)+1}{M^{2} n}\right] \\
& \leq c n^{d / 2}\left[\sqrt{t-s}+n^{-1 / 2}\right] .
\end{aligned}
$$

Similarly,

$$
\sum_{m \in \mathbb{Z}^{d}} P\left(D_{m}\right) \leq c n^{d / 2}\left[\sqrt{t-s}+n^{-1 / 2}\right] .
$$

The proof of (4.7) holds for indicator functions without any modifications. 
Acknowledgements This paper is part of my $\mathrm{Ph} . \mathrm{D}$. thesis at the University of Wisconsin-Madison. I would like to thank my advisor, Prof. Timo Seppäläinen, for his guidance. I would also like to thank Prof. Tom Kurtz for pointing out the connection to stochastic partial differential equations and for several helpful discussions.

Open Access This article is distributed under the terms of the Creative Commons Attribution Noncommercial License which permits any noncommercial use, distribution, and reproduction in any medium, provided the original author(s) and source are credited.

\section{References}

1. Bojdecki, T., Gorostiza, L.G.: Langevin Equations for $\mathcal{S}^{\prime}\left(\mathbb{R}^{d}\right)$-Valued Gaussian Processes and Fluctuation Limits of Infinite Particle Systems. Probability Theory and Related Fields, vol. 73(2). Springer, Berlin (1986)

2. Dürr, D., Goldstein, S., Lebowitz, J.L.: Asymptotics of particle trajectories in infinite one-dimensional systems with collisions. Commun. Pure Appl. Math. 38, 573-597 (1985)

3. Ethier, S.N., Kurtz, T.G.: Markov Processes, Characterization and Convergence. Wiley, New York (1986)

4. Holley, R., Stoock, D.: Generalized Ornstein-Uhlenbeck processes and infinite particle branching Brownian motions. Publ. RIMS Kyoto Univ. 14, 741-788 (1978)

5. Kallianpur, G., Xiong, J.: Stochastic Differential Equations in Infinite-Dimensional Spaces. Institute of Mathematical Statistics, Hayward (1995)

6. Kipnis, C., Landim, C.: Scaling Limits of Interacting Particle Systems. Grundlehren der Mathematischen Wissenschaften [Fundamental Principles of Mathematical Sciences], vol. 320. Springer, Berlin (1999)

7. Kumar, R.: Space-time current process for independent random walks in one dimension. ALEA Lat. Am. J. Probab. Math. Stat. 4, 307-336 (2008)

8. Martin-Löf, A.: Limit theorems for the motion of a Poisson system of independent Markovian particles with high density. Probab. Theory Relat. Fields 34, 205-223 (1976)

9. Mitoma, I.: Tightness of probabilities on $C\left([0,1] ; \mathcal{S}^{\prime}\right)$ and $D\left([0,1] ; \mathcal{S}^{\prime}\right)$. Ann. Probab. 11, 989-999 (1983)

10. Seppäläinen, T.: Second-order fluctuations and current across characteristic for a one-dimensional growth model of independent random walks. Ann. Probab. 33, 759-797 (2005)

11. Sethuraman, S.: Diffusive variance for a tagged particle in $d \leq 2$ asymmetric simple exclusion. ALEA Lat. Am. J. Probab. Math. Stat. 1, 305-332 (2006) (electronic)

12. Sethuraman, S.: Superdiffusivity of occupation-time variance in 2-dimensional asymmetric exclusion processes with density $\rho=1 / 2$. J. Stat. Phys. 123, 787-802 (2006)

13. Sethuraman, S.: On diffusivity of a tagged particle in asymmetric zero-range dynamics. Ann. Inst. H. Poincaré Probab. Stat. 43, 215-232 (2007)

14. Sethuraman, S., Varadhan, S.R.S., Yau, H.-T.: Diffusive limit of a tagged particle in asymmetric simple exclusion processes. Commun. Pure Appl. Math. 53, 972-1006 (2000)

15. Yau, H.-T.: $(\log t)^{2 / 3}$ law of the two dimensional asymmetric simple exclusion process. Ann. Math. 159, 377-405 (2004) 